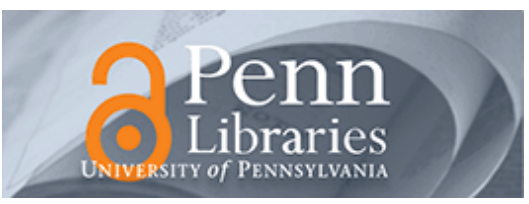

University of Pennsylvania

ScholarlyCommons

Departmental Papers (CIS)

Department of Computer \& Information Science

March 2003

\title{
A taxonomy and comparison of haptic actions for disassembly tasks
}

\author{
Aaron Samuel Bloomfield \\ University of Pennsylvania, aaronb@seas.upenn.edu \\ Yu Deng \\ University of Pennsylvania \\ Jeff Wampler \\ Air Force Research Laboratory, Deployment and Sustainment Division (AFRL/HES) \\ Pascale Rondot \\ GE Global Research \\ Dina Harth
}

See next page for additional authors

Follow this and additional works at: https://repository.upenn.edu/cis_papers

\section{Recommended Citation}

Aaron Samuel Bloomfield, Yu Deng, Jeff Wampler, Pascale Rondot, Dina Harth, Mary McManus, and Norman I. Badler, "A taxonomy and comparison of haptic actions for disassembly tasks", . March 2003.

Copyright (C) 2003 IEEE. Reprinted from Proceedings of the 2003 IEEE Virtual Reality Conference (VR'03), held 22-26 March 2003, pages 225-231. Publisher URL: http://ieeexplore.ieee.org/xpl/tocresult.jsp?isNumber=26695\&page=1 This material is posted here with permission of the IEEE. Such permission of the IEEE does not in any way imply IEEE endorsement of any of the University of Pennsylvania's products or services. Internal or personal use of this material is permitted. However, permission to reprint/republish this material for advertising or promotional purposes or for creating new collective works for resale or redistribution must be obtained from the IEEE by writing to pubs-permissions@ieee.org. By choosing to view this document, you agree to all provisions of the copyright laws protecting it.

This paper is posted at ScholarlyCommons. https://repository.upenn.edu/cis_papers/7

For more information, please contact repository@pobox.upenn.edu. 


\title{
A taxonomy and comparison of haptic actions for disassembly tasks
}

\begin{abstract}
The usefulness of modern day haptics equipment for virtual simulations of actual maintenance actions is examined. In an effort to categorize which areas haptic simulations may be useful, we have developed a taxonomy for haptic actions. This classification has two major dimensions: the general type of action performed and the type of force or torque required. Building upon this taxonomy, we selected three representative tasks from the taxonomy to evaluate in a virtual reality simulation. We conducted a series of human subject experiments to compare user performance and preference on a disassembly task with and without haptic feedback using CyberGlove, Phantom, and SpaceMouse interfaces. Analysis of the simulation runs shows Phantom users learned to accomplish the simulated actions significantly more quickly than did users of the CyberGlove or the SpaceMouse. Moreover a lack of differences in the postexperiment questionnaire suggests that haptics research should include a measure of actual performance speed or accuracy rather than relying solely on subjective reports of a device's ease of use.
\end{abstract}

\section{Keywords}

assembling, digital simulation, haptic interfaces, virtual reality, CyberGlove, Phantom, SpaceMouse interfaces, disassembly tasks, haptic actions, haptic feedback, haptic simulations, haptics research, human subject experiments, simulated actions, simulation runs, user performance, virtual reality simulation

\section{Comments}

Copyright @ 2003 IEEE. Reprinted from Proceedings of the 2003 IEEE Virtual Reality Conference (VR'03), held 22-26 March 2003, pages 225-231. Publisher URL: http://ieeexplore.ieee.org/xpl/

tocresult.jsp?isNumber $=26695 \&$ page $=1$

This material is posted here with permission of the IEEE. Such permission of the IEEE does not in any way imply IEEE endorsement of any of the University of Pennsylvania's products or services. Internal or personal use of this material is permitted. However, permission to reprint/republish this material for advertising or promotional purposes or for creating new collective works for resale or redistribution must be obtained from the IEEE by writing to pubs-permissions@ieee.org. By choosing to view this document, you agree to all provisions of the copyright laws protecting it.

\section{Author(s)}

Aaron Samuel Bloomfield, Yu Deng, Jeff Wampler, Pascale Rondot, Dina Harth, Mary McManus, and Norman I. Badler 


\title{
A Taxonomy and Comparison of Haptic Actions for Disassembly Tasks
}

\author{
Aaron Bloomfield ${ }^{1}$, Yu Deng ${ }^{1}$, Jeff Wampler ${ }^{2}$, Pascale Rondot ${ }^{3}$ \\ Dina Harth, Mary McManus, Norman Badler ${ }^{1}$ \\ ${ }^{1}$ University of Pennsylvania, Center for Human Modeling and Simulation \\ ${ }^{2}$ Air Force Research Laboratory, Deployment and Sustainment Division (AFRL/HES) \\ ${ }^{3}$ GE Global Research \\ aaronb@cis.upenn.edu
}

\begin{abstract}
The usefulness of modern day haptics equipment for virtual simulations of actual maintenance actions is examined. In an effort to categorize which areas haptic simulations may be useful, we have developed a taxonomy for haptic actions. This classification has two major dimensions: the general type of action performed and the type of force or torque required. Building upon this taxonomy, we selected three representative tasks from the taxonomy to evaluate in a virtual reality simulation. We conducted a series of human subject experiments to compare user performance and preference on a disassembly task with and without haptic feedback using CyberGlove, Phantom, and SpaceMouse interfaces. Analysis of the simulation runs shows Phantom users learned to accomplish the simulated actions significantly more quickly than did users of the CyberGlove or the SpaceMouse. Moreover a lack of differences in the post-experiment questionnaire suggests that haptics research should include a measure of actual performance speed or accuracy rather than relying solely on subjective reports of a device's ease of use.
\end{abstract}

\section{Introduction}

One particular application area that seems to be a natural testbed for haptic interaction is in validating disassembly or maintenance instructions. Since instructions are authored for human maintainers, reducing overall difficulty, avoiding errors, and improving safety should reduce costs and improve productivity. The prospect of using haptic simulation to aid in validating maintenance tasks led us to an analysis of possible tasks and ways to provide credible virtual task experiences.

Maintenance instructions provide a concrete application with verifiable results for haptic analysis. Real physical systems must be disassembled and repaired by maintenance technicians. Design decisions that impact maintainability can incur major cost over the lifetime of a complex system such as a modern aircraft. Validation means establishing that a given maintenance task could indeed be performed. Validating maintenance tasks in a virtual environment may help designers to create more maintainable systems, and can also serve to train technicians without taking an aircraft off the flight line or subjecting its components to the extra wear and tear of task practice.

In a technology investment agreement titled Service Manuals Generation (SMG), the Air Force Research Laboratory, Deployment and Sustainment Division (AFRL/HES) and GE Global Research are developing an automated technical manual development capability with a haptics enabled virtual validation environment. The SMG program is revolutionizing the way technical manuals are developed and providing a unique opportunity to validate the manuals before the system is built. Our research is directly supporting the SMG effort by evaluating various input devices across representative maintenance tasks.

The overall goal of this study is to investigate and compare virtual task validation with and without haptic feedback. There are four general methods to test and validate task performance:

1. Physical performance of task in real environment

2. Interactive computer user with visual feedback only

3. Interactive computer user with visual and haptic feedback

4. Non-interactive computational task analysis

Case 1 involves human action in a real system or fullscale mock-up. Cases 2 and 3 are investigated here using three different devices. Case 4 uses computation alone, such as a robotics reachability analysis and digital human mod- 
els, and is not presently feasible (primarily due to human variability) for complex situations.

In any of these cases there are four possible general outcomes:

A. The task is physically possible and humanly possible by any "typical" maintainer.

B. The task is physically possible but unreasonable to expect from a "typical" maintainer (e.g., insufficient strength).

C. The task is physically impossible due to human limitations, e.g., size (of any maintainer).

D. The task is physically impossible due to physical limitations (part is just inaccessible or not extractable).

To make the analysis tractable we elected to study only Cases 2 and 3 with a known performable task sequence (A). Testing for necessary strength or planning complex maneuvers around obstacles is beyond the scope of this inquiry. We accommodated tool use because it is a critical part of most maintenance actions.

In our study we used three commercially available interactive devices. The first was the Phantom, manufactured by SensAble Technologies, which is a device consisting of a rod attached to a robotic arm that is capable of providing force feedback. The second device was a CyberGlove, manufactured by Immersion Corporation, that can detect the exact position of the fingers. Although we have the CyberGrasp force feedback option for the CyberGlove, we did not use it as the forces it could apply would only affect finger movements, and fine motor control was not the experimental issue in our study. The third device was a SpaceMouse, manufactured by 3D Connexion, which is a hand-held 6 degree-of-freedom mouse-like device. The simulations ran on a high-end PC (Pentium 4, $2 \mathrm{GHz}$ ) workstation with an nVidia 3D graphics board.

This paper is organized as follows. First we describe background material on action taxonomy and how that relates to haptic actions. Then we give a physics-based action taxonomy and use this to select representative tasks for visual and haptic simulation. The experiment environment and protocol are given, followed by the experimental results and statistical analysis. We conclude with a discussion and suggestions for future work.

\section{Related Work}

Venkataraman and Iberall [14] provide a summary of grasps of the human hand. One of the earliest grasp taxonomies was summarized by Taylor and Schwarz [12] from a taxonomy originally defined by Schlesinger [10]. They describe six different grasps of the hand: cylindrical, fingertip, hook, palmar, spherical, and lateral. Cutkosky and
Wright $[5,6]$ defined a hierarchical tree structure taxonomy for grasps. This tree, while complete for maintenance tasks, is not exhaustive of all possible hand grasps; for example, holding a cigarette between the index and middle fingers is not provided by this model. The focus of both of these taxonomies is on how a hand grasps an object, and not necessarily on the action such a grasp may enable or require. Srinivasan and Basdogan [11] discuss haptic taxonomies, but their article is focused more on categorizing current haptics research. There are many other taxonomies that have been presented in research; due to space limitations, we can not reference them all.

There has been a lot of research in the area of comparing computer input devices. Card et. al. [4] evaluated a mouse, keyboard, and joystick for text selection on a CRT. Burdea [3] and Hayward and Astley [7] compare multiple haptic devices, as well as provide criteria for comparing them. These comparisons are based on measurements of key features (DOFs, motion range, peak force, etc.), and not experimental results. Payette et. al. [9] describe the experimental testing of the Pantograph (a custom haptic device) versus a trackball in zero-gravity and regular gravity, and presents experimental results in that article.

Significant research has been done in the area of virtual reality assembly and disassembly environments, in particular VADE (Virtual Assembly Design Environment) by Jayaram et. al. [8]. Our research is builds upon previous research done at the University of Pennsylvania [1, 2], which also focused on disassembly environments for aircraft maintenance.

\section{Haptic Action Taxonomy}

The range of actions we describe is not meant to be an exhaustive list of all possible human actions. Instead, it is a general classification of manual actions to be simulated in a virtual environment with haptics. These actions are mostly arm and hand actions, as that is what the majority of current haptic research, as well as current available haptic devices, focuses on. The classification consists of actions requiring fine motor control, significant arm strength, tactile friction, cooperative two-handed tasks, braced two-handed tasks, manipulating a deformable object, tool-assisted tasks, and multiple finger tasks. We characterize each of these below.

Fine motor control: These are actions that require very fine finger movements. Examples include pushing a pin, turning a bolt, or inserting a bayonet connector ${ }^{1}$. Not all haptic devices have the positional accuracy to properly simulate these tasks. While it is (relatively) easy to place a motion tracker on various body parts to know where it is

\footnotetext{
${ }^{1}$ A bayonet connector operates like the cap of a medicine bottle - you must first apply force to push the cap in, rotate it while the force is being applied, then release the force to remove the cap.
} 
positioned, the position of the fingers requires specialized equipment.

Significant arm strength: These tasks include any action that requires a significant amount of arm strength to perform. Examples include pulling open a stuck access panel, sanding (where considerable pressure must be placed on the sanding block), or pushing open a heavy door. Due to device limitations on the haptic devices, these tasks can often not be realistically simulated. The best most haptic devices can do is to exert a scaled-down force so that the user understands that a significant amount of force is required, while actually exerting less force.

Tactile (finger pressure) friction: These actions include anything that requires significant tactile feedback. A primary purpose of haptics is to provide touch and force feedback, but in these actions touch dominates. One can push open a door without haptic feedback, although the haptic feedback makes it feel more realistic. Examples include pushing a button, loading grease into a hole, or dialing a rotary-type telephone.

Cooperative two-handed tasks: These tasks include those actions that require the two hands to cooperate to achieve the desired effect. Examples include pushing two connectors together, using a two-handed tool, or lifting a bulky object. Note that the actions that each hand performs might fit into another category as well, and may fit into different categories. These actions require multiple haptic devices, along with the necessary computer support equipment to operate them. This often makes simulating these tasks difficult.

Braced two-handed tasks: These tasks require one hand to brace itself against an object while the other performs the action. Examples include holding a support while using a tool, or bracing one hand against a wall to pull open a door. The task performed with the one hand is often a task requiring significant arm strength (as if it were not, it would not require bracing). These tasks are much easier to simulate than the cooperative two-handed tasks, as one does not need a separate haptic device on the bracing hand. Only a rigid prop for the bracing hand is needed, though placement of the prop will need to be customized to the geometric configuration of the task.

Manipulating a deformable object: This category includes any tasks where the object being used is not a rigid body. Examples include wiping up a liquid spill, wringing out a towel, and twisting wires.

Tool assisted tasks: These are tasks that require a one handed tool to complete. Examples include using a hammer, screwdriver, crowbar, or wrench. These tasks are often easier to simulate with haptic devices, as the shaft of the haptics device that the user grasps naturally simulates the grasping of the tool shaft.
Multiple finger tasks: These require more than one finger to perform. Examples include grasping any object, pulling a pin by its head, or turning a bolt or dial. Note that some of these tasks can be simulated in the fine motor control category, above. However, that category does not necessarily take into account the interaction between the fingers.

\subsection{Forces and Torques}

We categorized force and torque requirements for haptic simulation as follows.

- Force Only I is a force direction aligned with the motion, such as pushing a door.

- Force Only II is a force direction not aligned with the motion, such as sanding with a block of wood.

- Torque Only I is a torque axis through grip space, such as using a screwdriver.

- Torque Only II is a torque axis that is not through grip space, such as using a lever device (i.e., a wrench) for leverage, or turning a steering wheel.

- Force and Torque is a broad category containing any action that requires both force and torque to complete, such as using a bayonet connector.

A couple of potential categories were omitted or combined. Requiring no force or torque is not particularly interesting and was omitted. The last category, where both force and torque are required, could have been further subdivided into four areas (each of the two force types with each of the two torque types), but we opted to combine them into one.

The haptic action task taxonomy is shown in table 1. The taxonomy helped determine which actions could be profitably simulated and compared in a virtual disassembly environment. We focused on the combination force and torque tasks to provide both utility and challenge to the experiment subjects. Everyone has had experience with both screwdrivers and bayonet connectors (such as a childproof medicine bottle cap), yet the user's actions are mostly dictated by the physics (forces and torques) and geometry (size, shape, turning angle) of the object. Our hypothesis is that the execution of those actions in a virtual environment would benefit from haptic feedback; that is what we sought to test. Since combinations of force and torque ought to be more complex than either alone, were we to detect no significant benefits to haptic feedback it would be unlikely that benefits would accrue for simpler cases. Conversely, were we able to detect benefits for haptics, we would expect simpler cases to inherit similar benefits.

\section{Experimental Procedure}

The virtual reality simulation that forms the basis for this experiment consisted of a series of actions the participants 


\begin{tabular}{|c|c|c|c|c|c|}
\hline & Force Only I & Force Only II & Torque Only I & Torque Only II & Force \& Torque \\
\hline $\begin{array}{l}\text { Fine motor } \\
\text { control }\end{array}$ & Pushing a pin & Wiping off grease & Turning a dial & $\begin{array}{l}\text { Using an } \\
\text { X-wrench }\end{array}$ & $\begin{array}{l}\text { Inserting a } \\
\text { bayonet } \\
\text { connector }\end{array}$ \\
\hline $\begin{array}{l}\text { Significant } \\
\text { arm strength }\end{array}$ & $\begin{array}{l}\text { Pulling open a } \\
\text { stuck access } \\
\text { panel }\end{array}$ & Sanding & Turning a stuck & $\begin{array}{l}\text { Using a wrench; } \\
\text { turning a crank }\end{array}$ & $\begin{array}{l}\text { Pushing a heavy } \\
\text { door while turn- } \\
\text { ing a lever latch }\end{array}$ \\
\hline $\begin{array}{l}\text { Tactile (finger } \\
\text { pressure) } \\
\text { friction }\end{array}$ & Pushing a button & $\begin{array}{l}\text { Loading grease } \\
\text { into a hole }\end{array}$ & $\begin{array}{l}\text { Inserting a small } \\
\text { bolt }\end{array}$ & $\begin{array}{l}\text { Dialing a } \\
\text { rotary-type phone }\end{array}$ & $\begin{array}{l}\text { Inserting a } \\
\text { bayonet } \\
\text { connector }\end{array}$ \\
\hline $\begin{array}{l}\text { Cooperative } \\
\text { two-handed } \\
\text { tasks }\end{array}$ & $\begin{array}{l}\text { Lifting a bulky } \\
\text { object; Pushing } \\
\text { two connectors } \\
\text { together }\end{array}$ & $\begin{array}{l}\text { Filing with a } \\
\text { large file }\end{array}$ & $\begin{array}{l}\text { Extracting a large } \\
\text { threaded rod or } \\
\text { bolt }\end{array}$ & $\begin{array}{l}\text { Dumping a } \\
\text { wheelbarrow load }\end{array}$ & $\begin{array}{l}\text { Pulling and } \\
\text { twisting a piston } \\
\text { from a cylinder }\end{array}$ \\
\hline $\begin{array}{l}\text { Braced } \\
\text { two-handed } \\
\text { tasks }\end{array}$ & \multicolumn{5}{|c|}{ Holding a support while doing a significant arm strength task } \\
\hline $\begin{array}{l}\text { Manipulating } \\
\text { a deformable } \\
\text { object }\end{array}$ & $\begin{array}{l}\text { Pushing to create } \\
\text { a shape (fuel } \\
\text { bladder removal) }\end{array}$ & $\begin{array}{l}\text { Holding a cloth } \\
\text { steady as it flaps } \\
\text { in the wind }\end{array}$ & Wringing a towel & $\begin{array}{l}\text { Stirring a viscous } \\
\text { liquid }\end{array}$ & $\begin{array}{l}\text { Twisting wires } \\
\text { while pulling the } \\
\text { cable taut }\end{array}$ \\
\hline $\begin{array}{l}\text { Tool-assisted } \\
\text { tasks }\end{array}$ & $\begin{array}{l}\text { Interface to } \\
\text { increase force per } \\
\text { unit area } \\
\text { (hammer, chisel) }\end{array}$ & $\begin{array}{l}\text { Interface to over- } \\
\text { come friction or } \\
\text { to increase force } \\
\text { per unit area } \\
\text { (plane, crowbar) }\end{array}$ & $\begin{array}{l}\text { Interface to } \\
\text { increase torque } \\
\text { (hex screwdriver) }\end{array}$ & $\begin{array}{l}\text { Interface to } \\
\text { increase torque } \\
\text { via leverage } \\
\text { (wrench) }\end{array}$ & $\begin{array}{l}\text { Interface to } \\
\text { increase torque } \\
\text { and force per unit } \\
\text { area (screwdriver) }\end{array}$ \\
\hline $\begin{array}{l}\text { Multi-finger } \\
\text { tasks }\end{array}$ & $\begin{array}{l}\text { Pushing multiple } \\
\text { buttons at once }\end{array}$ & $\begin{array}{l}\text { Pulling a pin by } \\
\text { its head }\end{array}$ & Turning a dial & $\begin{array}{l}\text { Turning a large } \\
\text { wing nut }\end{array}$ & $\begin{array}{l}\text { Inserting a } \\
\text { bayonet } \\
\text { connector }\end{array}$ \\
\hline
\end{tabular}

Table 1. Maintenance Task Taxonomy

had to perform to complete a disassembly task. The actions were: using a screwdriver (tool-assisted, torque I), using a wrench (tool-assisted, torque II), and removing a bayonet connector (fine motor control, force \& torque, although there are other categories it could fit in). The virtual reality simulation chained multiple instances of these actions together to create the disassembly task.

The scenario we used for our experiments was part of the removal of a fuel tank from an F-16 aircraft. As the purpose of our research was to evaluate the three devices, and to use a range of actions from the taxonomy, accuracy to the real task was not necessary. Thus, the simulation was based loosely on the actual Air Force Technical Order (maintenance instructions) for the removal of the fuel tank [13].

The simulation procedure was the same for all three devices. The tools operate the same way for all three platforms, but their appearance was dictated by the particulars of each device.
Figure 1 shows an image from the start of a simulation for the SpaceMouse. A screwdriver is on the upper left, and a wrench is on the upper right. The simulation consisted of 9 consecutive actions. The first two actions were the removal of the two screws holding the access panel shut. After the screws were removed, the access panel opened automatically.

The next action for the subject to perform was colored red, in order to indicate to the subjects which action was to be done when. This was done because the purpose of this research was to evaluate the different devices, and not to test the subject's memory of remembering the order of the actions.

Figure 2 shows an image from a CyberGlove simulation after the access panel was opened. The third task, colored red, was the removal of the bolt on the lower left side of the tank (in an actual F-16, this would drain the tank). The next three actions dealt with removing the three tubes that 


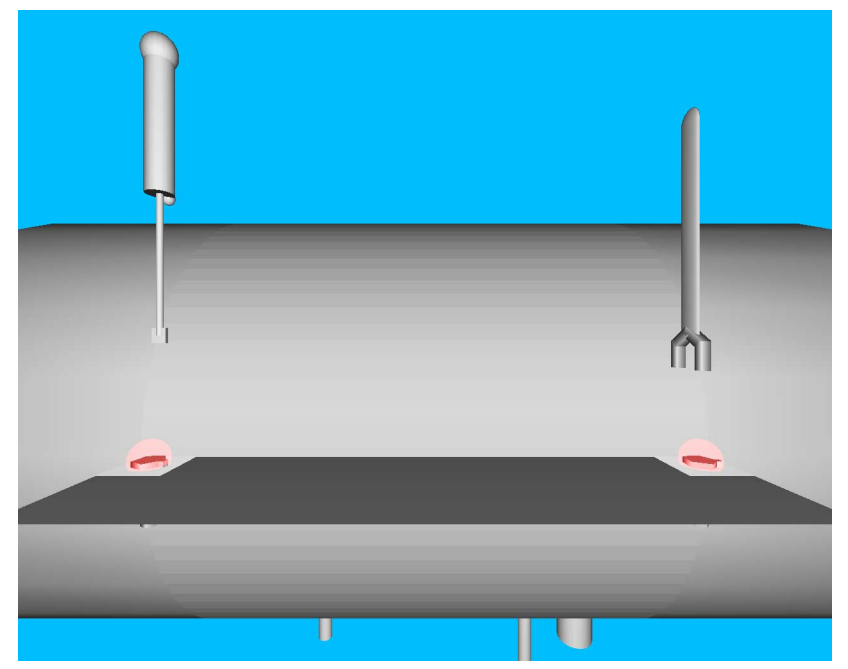

Figure 1. The initial view of the simulation for the SpaceMouse

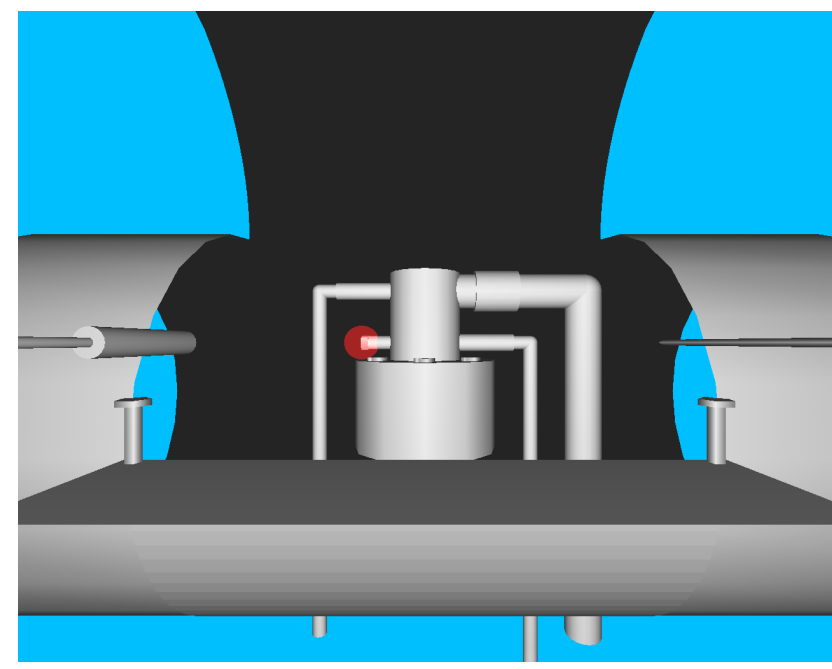

Figure 2. Partially completed simulation for the CyberGlove

connected to the fuel tank. The coupling on the upper right side of the tank was removed next, also with a wrench. The elbow attached to the coupling rotated automatically once this coupling was removed. The next two actions were the removal of bayonet connectors, the first on the lower right of the tank, and the second on the upper left of the tank. The last actions were the removal of the three screws that held the tank on the base.

Figure 3 shows a completed simulation for the Phantom. The base of the tank slides downward to indicate that the

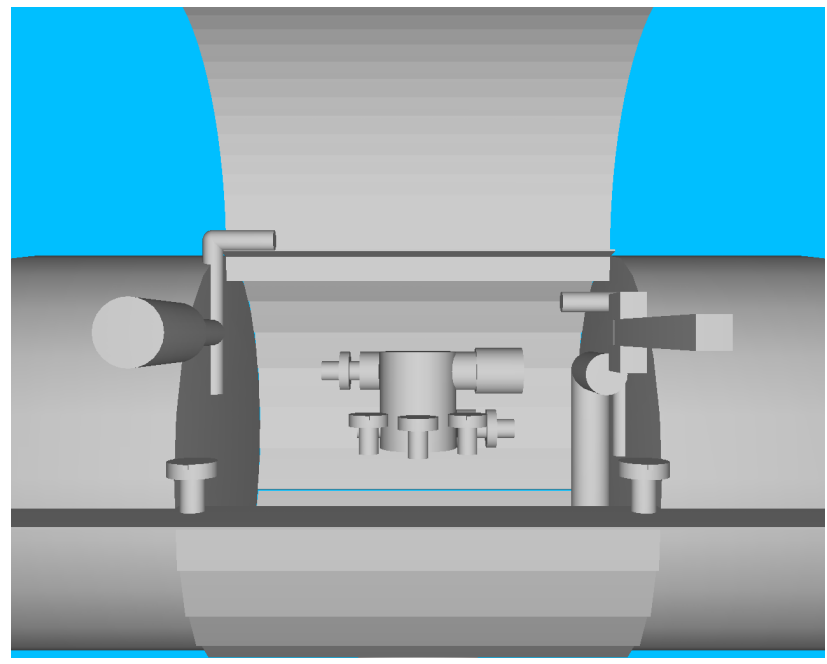

Figure 3. Completed simulation for the Phantom

simulation has been completed. The removal of the last three screws is not in the original technical order, but was included to provide the feeling of completing the task of removing the fuel tank. These last three screws were not included in the data analysis (the first two screws were, however).

Each participant ran the simulation on one of the three platforms. The subjects were arbitrarily assigned to a device. After signing the consent form, the subject viewed two videos, one that described the purpose of this research and what they were about to do, and the second which described how to use the particular device they were assigned. The subjects then proceeded to run through the simulation a total of six times. The first time was with an experimenter helping, to guide them through the process. Experimenter input was also permitted in the second simulation, if questions arose. For consistency across subjects, experimenter input consisted of repetition of relevant text from the video. Following all six simulation runs, they filled out a postexperiment questionnaire in which they rated how easy or difficult it was to perform the simulation using the particular device to which they were assigned.

\subsection{Accuracy}

In order for a participant to manipulate an object (such as a bolt) with a tool (such as a hex screwdriver), the tip of the tool had to be moved to within a certain distance of the object. This distance is represented by a sphere surrounding the object, and is shown to the user as a red translucent sphere, as shown in figures 1 and 2. As an aid to the user, 
the sphere would turn yellow when the tool tip was within the sphere, and green when the object's manipulation was completed.

The size of these spheres determined the level of accuracy for the simulation. A larger sphere would make it easier to complete the task, yet lower realism (the screwdriver could be far away from the bolt and still manipulate it). The sizes of the spheres were determined by informal experiments performed during development. The Phantom had the highest precision, and had the smallest sphere radius. The CyberGlove had the lowest precision, and thus had the largest sphere radius.

\section{Statistics and Experimental Measures}

The main data point used for statistical analysis is the sum of the times taken for the individual actions. This measurement is the main dependent variable because it takes into account mistakes made and corrective steps taken (i.e., you can't move to the next step without accurately completing the previous one, so mistakes cause an increase in time). The completion time does not include the time between steps (i.e., finding and grabbing the tool) that is variable across the devices.

For each simulation, the times for the individual actions were summed. These sums were then averaged across the last four simulations. The first two simulations were excluded from analysis because they served as "training" runs, with experimenter input. A one-way analysis of variance (ANOVA) was performed to determine whether statistically significant differences existed among the three devices in terms of this average time.

Ratings from the questionnaire were entered into a separate ANOVA to examine differences in perceived ease of use of the devices. The question was rated on a scale of 1 to 7.

To ensure equivalence of subject variables across groups, demographic data were analyzed via ANOVA or chi-square tests. These data included age, gender, and number of hours spent using a computer mouse in an average week.

All analyses were run with a level of significance set at $p<.05$.

\section{Experimental Results}

39 subjects participated in the study (average age 23.1; sd 4.9; 19 female, 20 male). There were 13 subjects arbitrarily assigned to each device. Groups did not differ statistically in terms of age or gender, nor in the amount of time spent using a computer mouse during an average week.

Analysis of the average speed across the last 4 simulations showed that performance speed was significantly lower (i.e. faster) for the Phantom than for the other two devices $[F(2,36)=5.699, p=.007]$. Average performance speeds were 58.0, 89.3, and 96.8 seconds for the Phantom, SpaceMouse, and CyberGlove, respectively. These results are shown in table 2 .

\begin{tabular}{|c|c|c|}
\hline Device & $\begin{array}{c}\text { Average } \\
\text { (seconds) }\end{array}$ & $\begin{array}{c}\text { Standard Deviation } \\
\text { (seconds) }\end{array}$ \\
\hline \hline SpaceMouse & 89.27 & 43.01 \\
Phantom & 57.98 & 23.35 \\
CyberGlove & 96.81 & 22.48 \\
\hline
\end{tabular}

Table 2. Mean Performance Speed per Device

Post-hoc analyses of the time taken confirmed that significant differences existed between the Phantom group and the other groups, but not between the CyberGlove and SpaceMouse groups. These results are shown in table 3.

\begin{tabular}{|c|c|c|c|c|}
\hline \multicolumn{2}{|c|}{ Groups } & $\begin{array}{c}\text { Mean } \\
\text { Difference }\end{array}$ & $\begin{array}{c}\text { Standard } \\
\text { Error }\end{array}$ & Significance \\
\hline \hline SM & PH & 31.29 & 12.20 & 0.038 \\
& CG & -7.54 & 12.20 & 0.811 \\
\hline PH & SM & -31.29 & 12.20 & 0.038 \\
& CG & -38.83 & 12.20 & 0.008 \\
\hline CG & SM & 7.54 & 12.20 & 0.811 \\
& PH & 38.83 & 12.20 & 0.008 \\
\hline
\end{tabular}

Table 3. Results of Tukey Post-Hoc Analysis

An additional ANOVA showed that ratings on perceived ease of use of the device did not differ significantly among groups.

\section{Conclusions}

Results show that the Phantom users learned to accomplish the simulated actions significantly more quickly than did users of the CyberGlove or the SpaceMouse. This could translate into lower training costs and/or more efficient training sequences. The 3D graphics simulations and the user's ability to directly manipulate objects were comparable, so we attribute the decreased performance times to two main factors.

The Phantom differs from the other two devices in several ways, including its use of haptic feedback. It is possible that the haptic feedback contributes to faster performance, although a more direct comparison of haptic-feedback vs. no-haptic-feedback (both using the Phantom) would offer more rigorous assessment of this variable. 
The other factor is the increased precision that is inherent in the Phantom. The range of motion for the Phantom is limited to a specific volume (the range of the Phantom arm), which inherently maps onto the virtual world. By comparison, the SpaceMouse and CyberGlove users had more difficulty moving to a specific location in the simulation.

The lack of differences in the post-experiment questionnaire suggests that future research should include a measure of actual performance speed and/or accuracy (whether in simulations, or in real life tasks), rather than relying solely on subjective reports of a device's ease of use.

\section{Future Work}

The haptics taxonomy provided a framework for examining maintenance actions. Our results implied an execution time advantage for users executing combined force and torque tasks using the Phantom over non-haptics CyberGlove and SpaceMouse devices.

Generalizing this result, it is possible that complex tasks could benefit from human feasibility analysis using haptics simulation and thus that selected task simulations can assist in developing valid service manuals. The primary moderating factor in our experiments was the relatively low levels of force and torque reacted through the Phantom. As users were more successful at simulated tasks using haptic feedback, it is possible that more strenuous challenges would benefit from similar feedback, but this would need to be tested in a more "industrial-strength" haptics feedback environment. The difficulty here is ensuring the safety of the subjects.

Recall that we categorized virtual task performance four ways:

1. Physical performance of task in real environment

2. Interactive computer user with visual feedback only

3. Interactive computer user with visual and haptic feedback

4. Non-interactive computational task analysis

Significant benefits would accrue to system and task designers if the fourth option were realizable. Obtaining geometric models of aircraft systems is not too difficult, and digital human models are commercially available. But our experiments seem to show that task performance depends on haptic feedback in a way that no existing computational model can accommodate. Embedding human performance capability models into kinematic human models seems to be a necessary and desirable step toward automating the analysis of maintenance tasks for service manual generation and validation.

\section{Acknowledgments}

This research is partially supported by Air Force F33615-99-D-6001 Delivery Order \#8 and NASA 00HEDS-01-052.

\section{References}

[1] N. Badler, R. Bindiganavale, C. Erignac, and P. Vincent. Technology assessment for automated technical order validation. Technical report, Air Force Research Laboratory, Deployment and Sustainment Division (AFRL/HES), 2000.

[2] N. I. Badler, C. A. Erignac, and Y. Liu. Virtual humans for validating maintenance procedures. Communications of the ACM, 45(7):56-63, July 2002.

[3] G. C. Burdea. Force and Touch Feedback for Virtual Reality. John Wiley and Sons, New York, 1996.

[4] S. Card, W. K. English, and B. J. Burr. Evaluations of mouse, rate-controlled isometric joystick, step keys, and text keys for text selection on a CRT. Ergonomics, 21(8):601613, Aug. 1978.

[5] M. R. Cutkosky. On grasp choice, grasp models, and the design of hands for manufacturing tasks. IEEE Journal of Robotics and Automation, 5(3):269-279, June 1989.

[6] M. R. Cutkosky and P. K. Wright. Modeling manufacturing grips and correlations with the design of robotic hands. In Proceedings of the IEEE International Conference on Robotics and Automation, pages 1533-1539, 1986.

[7] V. Hayward and O. R. Astley. Performance measures for haptic interfaces. In G. Giralt and G. Hirzinger, editors, Robotics Research: The 7th International Symposium, pages 195-207, 1996.

[8] S. Jayaram, U. Jayaram, Y. Wang, H. Tirumali, K. Lyons, and P. Hart. VADE: A virtual assembly design environment. IEEE Computer Graphics and Applications, 19(6):44-50, Nov./Dec. 1999.

[9] J. Payette, V. Hawyard, C. Ramstein, and D. Bergeron. Evaluation of a force feedback (haptic) computer pointing device in zero gravity. In Proceedings of the ASME Dynamcis Systems and Control Division, volume 58, pages 547-553, 1996.

[10] G. Sclesinger. Der mechanische aufbau der kunstlichen glieder (the mechanical building of artifical limbs). In M. Borchardt, editor, Ersatzglieder und Arbeitshilfen fur Kriegsbeschadigte und Unfallverletzte (Prosthetic and Occupational Help for War Victims and Accident Victims), pages 321-699. Springer, 1919.

[11] M. A. Srinivasan and C. Basdogan. Haptics in virtual environments: taxonomy, research status, and challenges. Computers and Graphics, 21(4):393-404, July-Aug. 1997.

[12] C. L. Taylor and R. J. Schwarz. The anatomy and mechanics of the human hand. In Artifical Limbs, 2, pages 22-35, 1955.

[13] United States Air Force. Technical Order $1 F-16 C G-2-28 J G-$ 20-1. Figure 2-14-1: Removal of Internal Fuel Tank Vent and Pressurization Valve.

[14] S. T. Venkataraman and T. Iberall. Dextrous Robot Hands. Springer-Verlag, New York, 1988. 